\title{
COLLECTIVE ELECTRON-POSITRON ANNIHILATION
}

\author{
A. A. Belyanin, V. V. Kocharovskit, and Vl. V. Kocharovskit \\ Institute of Applied Physics, Academy of Sciences
}

\begin{abstract}
The phenomenon of collective spontaneous annihilation of a magnetized electron-positron plasma is predicted. Like the superradiance in systems with discrete energy spectra, collective annihilation leads to the generation of powerful coherent radiation with the rate of this process considerably exceeding the spontaneous annihilation and collisional relaxation rates.
\end{abstract}

1. Annihilation processes in electron-positron $\left(e^{-} e^{+}\right)$plasmas and their various applications are of great interest. The traditional treatment of both spontaneous and induced annihilation is based on the quantum-electrodynamic calculation of S-matrix terms and solution of the rate equations for pair and photon densities. However, we have found that annihilation in a sufficiently dense $e^{-} e^{+}$plasma demonstrates collective, coherent behavior with the rate of the process considerably exceeding the incoherent relaxation rates. As a result, a pulse of coherent $\gamma$-radiation is generated with spectral power density much greater than that for annihilation of the same number of independent pairs. Such a manifestly nonstationary process cannot be described in terms of the rate equations and independent spectral components of the radiation due to correlations in the broad frequency band width $\Delta t^{-1} \gg T^{-1}$, where $\Delta t$ is the duration of the annihilation pulse, and $T$ is a characteristic relaxation time.

This paper is devoted to one of the possible variants of collective annihilation (CA): collective onequantum annihilation decay of a magnetized $e^{-} e^{+}$plasma at magnetic field strength $B$, where $B_{\mathrm{c}} \cong$ $4.4 \times 10^{13} \mathrm{G}$. We point out the straightforward analogy between such a decay and collective spontaneous emission (superradiance) in systems of excited (population-inverted) atoms or molecules. We present an example of an analysis of superradiance in a medium with a continuous energy spectrum. Superradiance has only been investigated in systems with discrete energy spectra [see, e.g. Gross and Haroche 1982, Andreev et al. (1988), Zheleznyakov et al. (1989), and Kocharovskii and Kocharovskii (1990)].

We assume the number of photons to be sufficiently large to use the classical Maxwell equations for the electromagnetic field. The magnetized $e^{-} e^{+}$plasma is described by the polarizability tensor $\chi_{i j}$ evaluated first in Svetozarova and Tsytovitch (1962). The covariant expression for $\chi_{i j}$ re-derived in Melrose and Parle (1983) will be used here.

It is known that the population inversion with respect to one-quantum annihilation in an $e^{-} e^{+}$plasma occurs when the $e^{-} e^{+}$pairs become degenerate. We shall confine ourselves for simplicity to completely degenerate $e^{-} e^{+}$pairs occupying the lowest Landau level $n=0$ with equal electron and positron densities:

$$
N_{\mathrm{e}} \equiv \frac{1}{2 \pi^{2} \lambda_{\mathrm{c}}^{9}} \frac{B}{B_{\mathrm{c}}} \frac{p_{\mathrm{F}}}{m c}<N_{1}=\left(\frac{B}{B_{\mathrm{c}}}\right)^{3 / 2} \frac{1}{\sqrt{2} \pi \lambda_{\mathrm{c}}^{9}} \cong\left(\frac{B}{B_{\mathrm{c}}}\right)^{3 / 2} 1.2 \times 10^{30} \mathrm{~cm}^{-3},
$$

where $\lambda_{c}=\hbar / m c$ is the electron Compton wavelength. The inequality implies that the Fermi energy

$$
\mu \equiv E_{n=0}\left(p_{z}=p_{F}\right)<E_{n=1}\left(p_{z}=0\right),
$$

where $p_{\mathrm{F}}$ is the Fermi momentum, and the energy of a Dirac electron in magnetic field $B=B z^{0}$ is given by $E_{n}=\left[p_{z}^{2} c^{2}+m^{2} c^{4}\left(1+2 n B / B_{c}\right)\right]^{1 / 2}$; see figure 1 . This allows us to treat the exited Landau levels as unoccupied.

2. First of all we find the normal modes in the homogeneous magnetized $e^{-} e^{+}$plasma. Nornal modes in such a medium were analyzed, e.g. by Shabad $(1975,1988)$. Since we are interested in the behavior of modes near the first one-quantum annihilation resonance $\omega=\omega_{0}$ [see eqs.(4) and (5) below] we shall neglect the contribution of excited Landau levels to $\chi_{i j}$. Then the integration over longitudinal momentum $p_{z}$ in $\chi_{i j}$ may be performed exactly, and the value of the hermitian part of $\chi_{i j}$ at different pair densities and its influence on the behavior of growth rate $\omega_{e}^{\prime \prime}(k)=\Im\left\{\omega_{e}(k)\right\}$ may be estimated. For sufficiently large pair densities [see eq.(7)] the behavior of the growth rate $\omega_{e}^{\prime \prime}(k)$ coincides with that obtained by Shabad. 


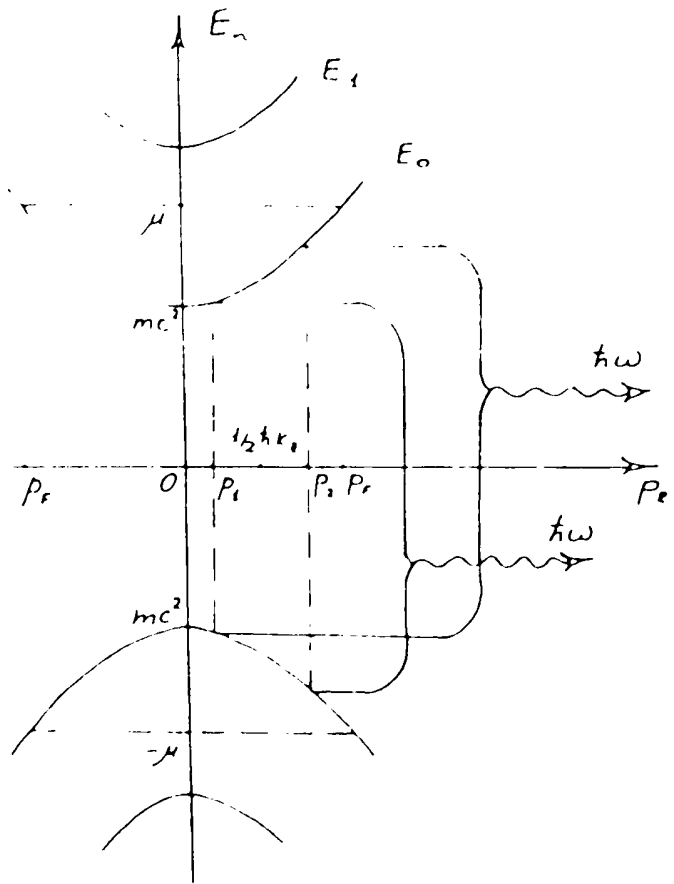

Figure 1a Energy-level diagram for one-quantum annihilation of Dirac electrons and positrons.

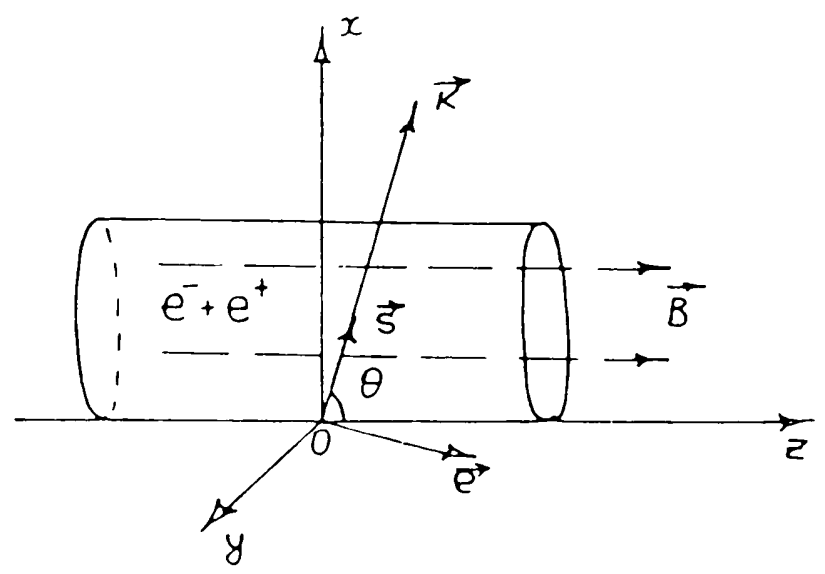

Figure 1 b Geometry of CA radiation of extraordinary $\gamma$ quanta with wave vector $\boldsymbol{k}$ and polarization $\boldsymbol{e}$ in the $e^{-} e^{+}$ plasma sample.

Choosing the coordinate frame as is shown in figure 1 , we obtain for the permittivity tensor $\varepsilon_{i j}=$ $\delta_{i j}+4 \pi \chi_{i j}$ with the only nonzero component of polarizability tensor

$$
\begin{aligned}
\chi_{z z}(\omega, k)= & \frac{\sqrt{2} m^{5} c^{9} b}{\pi^{2} \hbar^{4} \omega^{2}\left(\omega^{2}-c^{2} k_{z}^{2}\right)^{2} \beta}\left\{-\frac{4 \pi i \hbar \omega^{3}}{m c^{2}\left(\omega^{2}-c^{2} k_{z}^{2}\right)}\left[\eta\left(p_{F}-\left|p_{1}\right|\right)\right.\right. \\
& \left.\left.+\eta\left(p_{\mathrm{F}}-\left|p_{2}\right|\right)-1\right]-\frac{4 \hbar k_{z} \beta}{m^{3} c^{4}}\left(\mu_{+}-\mu_{-}\right)+\sum_{g=1}^{2} \frac{(-1)^{g} \varphi_{g} \mathcal{L}_{g}}{\left(1+\left(p_{g} / m c\right)^{2}\right)^{1 / 2}}\right\}
\end{aligned}
$$

where $\alpha=e^{2} / \hbar c$,

$$
\begin{aligned}
& b=\frac{\alpha}{8 \sqrt{2}} \frac{B}{B_{\mathrm{c}}} \exp \left[-\frac{2 B_{\mathrm{c}}}{B}\left(\frac{\hbar k \sin \theta}{2 m c}\right)^{2}\right], \beta=(\hbar \omega / 2 c)\left[1-4 m^{2} c^{4} / \hbar^{2}\left(\sqrt{\omega^{2}-c^{2}} k_{z}^{2}\right)\right]^{1 / 2}, \\
& \varphi_{1,2}=\left(\hbar \omega / m c^{2}\right)^{2}+\frac{\hbar k_{z}\left(\hbar k_{z} \pm 2 \beta\right) \omega^{2}}{m^{2} c^{2}\left(\omega^{2}-c^{2} k_{z}^{2}\right)}, p_{1,2}=\frac{1}{2} \hbar k_{z} \pm \beta, \mu=\left(m^{2} c^{4}+p_{F}^{2} c^{2}\right)^{1 / 2}, \\
& \mu_{ \pm}=\left[m^{2} c^{4}+\left(\hbar k_{z} \pm p_{\mathrm{F}}\right)^{2} c^{2}\right]^{1 / 2}, \eta(x)=\left\{\begin{array}{ll}
1, & x \geq 0 \\
0, & x<0
\end{array},\right. \\
& \mathcal{L}_{g}=\ln \left|\frac{\left(p_{\mathrm{F}}+p_{g}\right)\left(\hbar k_{z}-p_{\mathrm{F}}-p_{g}\right)\left[m^{2} c^{2}+p_{\mathrm{F}} p_{g}+\mu\left(m^{2}+p_{g}^{2} / c^{2}\right)^{1 / 2}\right]}{\left(p_{\mathrm{F}}-p_{g}\right)\left(\hbar k_{z}+p_{\mathrm{F}}-p_{g}\right)\left[m^{2} c^{2}-p_{\mathrm{F}} p_{g}+\mu\left(m^{2}+p_{g}^{2} / c^{2}\right)^{1 / 2}\right]}\right|+ \\
& \ln \left|\frac{\left[m^{2} c^{2}+\left(\hbar k_{z}+p_{\mathrm{F}}\right) p_{g}+\mu_{+}\left(m^{2}+p_{g}^{2}\right)\right]\left[\left(m^{2} c^{2}+p_{g}^{2}\right)^{1 / 2}-p_{g}\right]}{\left[m^{2} c^{2}+\left(\hbar k_{z}-p_{\mathrm{F}}\right) p_{g}+\mu_{-}\left(m^{2}+p_{g}^{2} / c^{2}\right)\right]\left[\left(m^{2} c^{2}+p_{g}^{2}\right)^{1 / 2}+p_{g}\right]}\right| \text {. }
\end{aligned}
$$

Note the singularity in eq.(3) at the threshold frequency of one-quantum annihilation at the lowest Landau level

$$
\omega_{0}^{2}=c^{2} k_{z}^{2}+\left(2 m c^{2} / \hbar\right)^{2} .
$$

The frequency range near $\omega=\omega_{0}$ determines the dynamics of the annihilation process. Near the resonance, using $\left|\omega-\omega_{0}\right| \ll \omega_{0}$ and replacing $k_{z} \rightarrow\left(\omega_{0} / c\right) \cos \theta$ everywhere in eq.(3) except resonant terms, this expression may be considerably simplified. After solving the dispersion relation 
$\omega^{2}=c^{2} k^{2}-4 \pi \chi_{z z}(\omega, k)\left(\omega^{2}-c^{2} k_{z}^{2}\right)$, we obtain that in the degenerate plasma the extraordinary mode with quasi-transverse polarization in the $(k, B)$ plane becomes unstable. The curves $\omega_{e}^{\prime}(k)=$ $\operatorname{Re}\left[\omega_{\mathrm{e}}(k)\right] a n d \omega^{\prime \prime}(k)=\Im\left\{\omega_{\mathrm{e}}(k)\right\}$ are shown in figure 2. The maximum growth rate is given by

$$
\omega^{\prime \prime} / \omega_{0} \cong(\sqrt{3} / 2)\left[(\alpha / 8 \sqrt{2})\left(B / B_{c}\right) \exp \left(-2 B_{c} / B\right)\right]^{2 / 3} \sin ^{2} \theta
$$

where $\omega_{0} \cong 2 m c^{2} / \hbar \sin \theta$ is assumed to be much greater $\operatorname{than} \omega^{\prime \prime}$. For $B=B_{c}, \theta=\pi / 2$ one has $\omega^{\prime \prime} \cong 3 \times 10^{18} \mathrm{~s}^{-1} \ll \omega_{0} \cong 2 \times 10^{21} \mathrm{~s}^{-1}$.

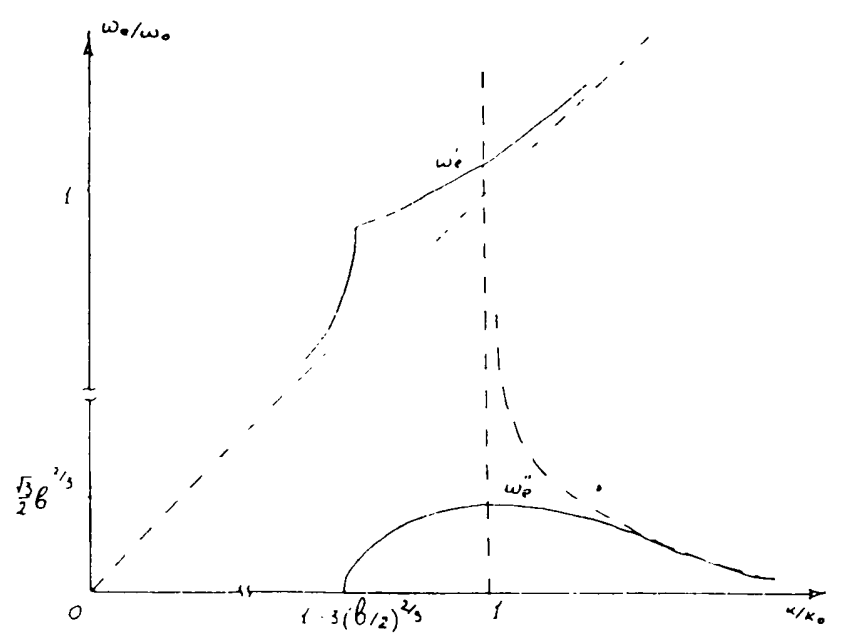

Figure 2 Dispersion curves for the unstable extraordinary mode $w_{e}(k)=w_{e}^{\prime}(k)+i \omega_{e}^{\prime \prime}(k)$ near the one-quantum annihilation resonance $\omega_{0} \equiv c k_{0}=2 m c^{2} / \hbar \sin \theta$ (solid line) and in the region $\left|\omega-\omega_{0}\right| \gg \omega^{\prime \prime}$ where $\left|\chi_{z z}(\omega=c k)\right| \ll 1$ and an iteration solution of dispersion equation becomes possible (dashed line).

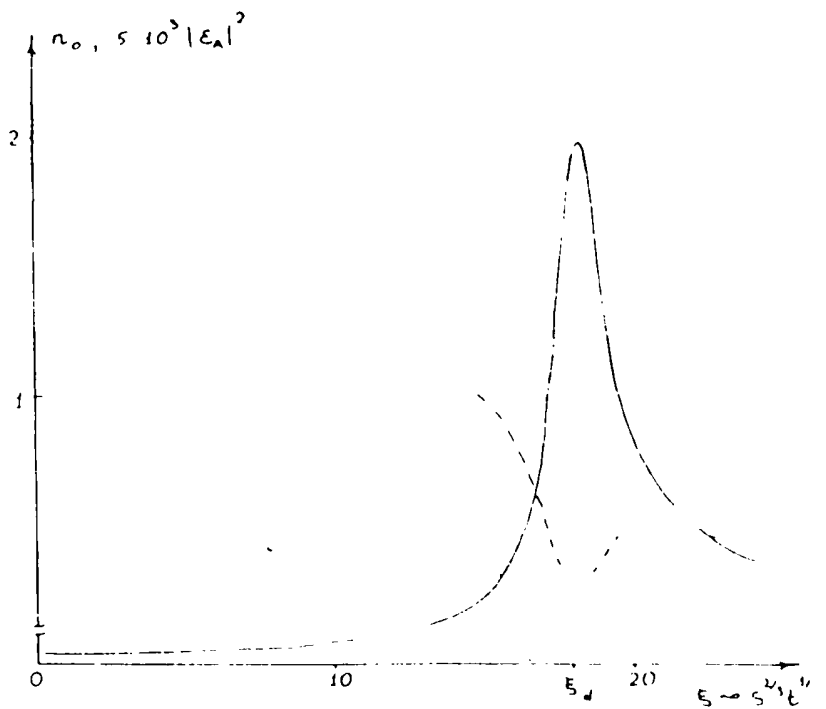

Figure 3 Self-similar profiles of field amplitude squared $\left|\varepsilon_{A}\right|^{2}$ (solid line) and pair occupation number $n_{0}$ (dashed line) according to eq.(14). The curves are plotted numerically for $\varepsilon_{\mathrm{A}}(0)=10^{5} ; n_{0}(0)=1$.

The scale of spatial coherency of the process is the cooperative length $L_{c}$-the maximum distance between pairs interacting by the field of their radiation up to $t \approx 1 / \omega^{\prime \prime}$, i.e. $L_{c} \approx c / \omega^{\prime \prime} ; L_{\mathrm{c}} \approx 1 \AA$ for $B=B_{\mathrm{c}}$, and $\theta=\pi / 2$.

One should note that the curves $\omega_{\mathrm{e}}^{\prime}(k)$ and $\omega^{\prime \prime}(k)$ are insensitive to variations in pair density. Moreover, the value of $\omega^{\prime \prime}$ depends only on the values of $B$ and $\theta$. Such saturation occurs only in a sufficiently dense plasma when all states in the $p_{z}$-interval

$$
\Delta p_{\mathrm{F}}=\hbar\left(\omega_{0} \omega^{\prime \prime}\right)^{1 / 2} \sin \theta / c
$$

around the value $p_{z}=\hbar k_{z} / 2$ are occupied by particles. In other words, the saturation occurs when $p_{\mathrm{F}} \downarrow\left(\Delta p_{\mathrm{F}}+\hbar k_{z}\right) / 2$, i.e.

$$
N_{\mathrm{e}} \downarrow N_{\mathrm{e}}^{(\mathrm{s})}=N_{0} \frac{B\left[\Delta p_{\mathrm{F}}+\hbar k_{z}\right]}{B_{\mathrm{c}} m c} \equiv N_{0} \frac{B}{B_{\mathrm{c}}}\left[\left(\frac{2 \hbar \omega^{\prime \prime}}{m c^{2} \sin ^{3} \theta}\right)^{1 / 2}+2 \cot \theta\right]
$$

$N_{0}=\left(4 \pi^{2} \lambda_{\mathrm{c}}^{3}\right)^{-1} \cong 4 \times 10^{29} \mathrm{~cm}^{-3}$. For transverse propagation one has $N_{\mathrm{e}}^{(\mathrm{s})}\left(B=B_{\mathrm{c}}\right) \cong 4 \times 10^{28} \mathrm{~cm}^{-3}$, and $N_{\mathrm{e}}^{(\mathrm{s})}\left(B=B_{\mathrm{c}} / 4\right) \cong 10^{27} \mathrm{~cm}^{-3}$.

3. Now let us consider the generation of annihilation radiation $e_{z}(t, s)=(1 / 2) \varepsilon_{z}(t, s) \exp \left(-i \omega_{0} t+\right.$ $\left.i k_{0} s\right)+$ c.c. with a given initial $(\mathrm{t}=0)$ level of spontaneous fluctuations $\varepsilon_{0}(s)$ and its propagation along one direction $s$ (figure $1 \mathrm{~b}$ ). At the linear stage when the pair density $N_{e}=$ const and the slowly changing field amplitude $\varepsilon_{z}(t, 8)$ is sufficiently small, one may obtain

$$
\left[c \partial / \partial s+p-2 \pi i \omega_{0} \chi_{z z}(p) \sin ^{2} \theta\right] \varepsilon_{z}(p, s)=\varepsilon_{0}(s),
$$

where $\left|\omega-\omega_{0}\right| \ll \omega_{0}, p=i\left(\omega_{0}-\omega\right)$ and $\varepsilon_{z}(p, s)=\int_{0}^{\infty} \varepsilon_{z}(t, s) \exp (-p t) d t$. An asymptotic $(\xi \gg 1)$ solution of eq. ( 8 ) is given by

$$
\varepsilon_{z}(t, s) \cong \varepsilon_{0}(0) \frac{4 \pi s}{3 c t}(3 / 2 \xi)^{1 / 2} \exp \left(\xi e^{-i \pi / 8}\right) ;
$$




$$
\xi=\left[(6 \sqrt{3})^{1 / 2} s / L_{\mathrm{c}}\right]^{2 / 3}\left(\omega^{\prime \prime} t\right)^{1 / 3} \gg 1 .
$$

To find the space-time profile of the radiation pulse one should take into account the nonlinear depletion of pair density. To describe this nonlinear stage qualitatively we suppose that the radiation along the direction $\theta$ is formed completely by annihilation of the group of pairs occupying an appropriate $p_{z}$-interval [eq.(6)] $\Delta p_{\mathrm{F}}(\theta)$ around $\hbar k_{z} / 2$. Then the different groups of pairs will annihilate independently, each forming a mode with appropriate $\omega_{0}$ and $k_{z}$. In this approximation, the evolution [averaged over $\Delta p_{\mathrm{F}}$ occupation number $n_{0}$ of electron (positron) states with energy $\left.E_{0} \cong \hbar \omega_{0} / 2\right]$ is determined by the energy conservation law

$$
\Delta N_{\mathrm{e}} \partial n_{0} / \partial t=\Im\left\{\mathcal{P} \varepsilon_{z}^{*}\right\} / 2 \hbar ; \quad \Delta N_{\mathrm{e}} \equiv N_{0} \frac{B}{B_{\mathrm{c}}} \frac{\Delta p_{\mathrm{F}}}{m c},
$$

where $\varepsilon$ and $\mathcal{P}$ are the field and polarization amplitudes. This equation together with the material equation

$$
\begin{gathered}
\mathcal{P}(t, s)=-\frac{b_{0}}{2 \pi} \sqrt{\omega_{0}} \sin \theta \exp (i \pi / 4) \int_{0}^{t} \frac{\left[2 n_{0}\left(t^{\prime}, s\right)-1\right] \varepsilon_{z}\left(t^{\prime}, s\right)}{\left[\pi\left(t-t^{\prime}\right)\right]^{1 / 2}} d t^{\prime} \\
b_{0} \equiv(\alpha / 8 \sqrt{2})\left(B / B_{\mathrm{c}}\right) \exp \left(-2 B_{\mathrm{c}} / B\right)
\end{gathered}
$$

and wave equation

$$
\partial \varepsilon_{z} / \partial t+c \partial \varepsilon_{z} / \partial s=2 \pi i \omega_{0} \mathcal{P} \sin ^{2} \theta
$$

describes the nonlinear $\mathrm{CA}$ regime. It may be shown that the system of equations (11)-(13) has the self-similar solution

$$
\varepsilon(t, s)=\varepsilon_{\mathrm{n} \mid} \varepsilon_{\mathrm{A}}(\xi)\left[(6 \sqrt{3})^{1 / 2} s / L_{\mathrm{c}}\right]^{3 / 2}, \quad 2 n_{0}(t, s)-1=\Delta n(\xi)
$$

where $\epsilon_{\mathrm{nl}}^{2}=\hbar \omega_{0} \sin ^{2} \theta \Delta N_{\mathrm{e}}$ and $\xi$ is given by eq. (10).

The solution [eq.(14)] gives universal profiles of $\varepsilon_{\mathrm{A}}(\xi)$ and $n_{0}(\xi)$ as shown in figure 3 . When $n_{0}=$ const, it agrees with eq.(9). According to eq.(9) the value of $\varepsilon(\xi)$ achieves the maximum level $\varepsilon_{\mathrm{m}}$ at the delay time $t_{\mathrm{d}}=\xi_{\mathrm{d}}^{3} /\left(6 \sqrt{3} \omega^{\prime \prime}\left(s / L_{\mathrm{c}}\right)^{2}\right)$, where $\xi_{\mathrm{d}} \cong \ln \left|\varepsilon_{\mathrm{m}} / \varepsilon_{0}\right| \gg 1$. When $B=B_{\mathrm{c}}$ and $s$ is of order $L_{\mathrm{c}}$, the value of $\varepsilon_{\mathrm{m}}$ is of order $3 \times 10^{-4} B_{\mathrm{c}}$.

The duration of the front of the pulse $\tau \cong 3 t_{d} / \xi_{d}$ is determined by eq.(9), while the duration of the whole CA pulse $\tau$ is defined by the annihilation rate of most of the pairs in the plasma sample. In other words, $\tau$ is determined by the movement of the depletion front $\xi=\xi_{\mathrm{d}}$ (see figure 3 ) from the plasma boundary deep into the sample. One may easily show that $\tau$ is of order $t_{\mathrm{d}}$. The values of the characteristic time scales are shown in figure 5.

We note that if the length of the sample $s$ is much greater than $c t_{d} \cong L_{c} \xi_{d} / 2$, the regular solutions eqs.(9) to (14) are no longer correct. In this case at the different segments $\sim c t_{d}$ independent CA pulses will be generated. Finally, their propagation and reabsorption in the plasma results in forming the irregular sequence.

We have investigated a one-dimensional model. It may be shown that the angular divergence of the radiation does not change the dynamics of the $C A$. It is caused by the fact that the waves propagating along different directions $s$ are mutually incoherent and amplified independently of each other. Therefore, the generation of $\gamma$-radiation is determined by the self-similar solution eqs.(9) and (14) along each direction $\boldsymbol{s}$.

4. Using the results obtained in sections 2 and 3 one can easily paint a qualitative picture of the CA of a real three-dimensional plasma bunch. For fairly short bunches $\left[L_{z} \ll c t_{d}\left(1+m^{2} c^{2} / p_{F}^{2}\right)^{-1 / 2}\right]$ one must take into account the expansion of independently annihilating groups of pairs (see the end of section 2) along the magnetic field lines. Flying away along the field lines the groups will annihilate along different directions $\theta$ determined by $\cos \theta=z / c t_{\mathrm{d}}$ (figure 4) at different frequencies $\omega_{0}(\theta)$. Due to the relative movement of pairs, the size of a certain group along the $z$-direction will be $\Delta L_{z} \cong 2 c t_{d}\left(\omega^{\prime \prime} / \omega_{0}\right)^{1 / 2} \sin \theta$. If the $z$-size of the initial plasma bunch is greater than $\Delta L_{z}$, we need not take into account the depletion of pair density caused by their relative movement, and all previous results may be applied to each group.

The power $\Delta \mathcal{W}$ of coherent $\gamma$-radiation for each group with sizes $L_{x} \sim L_{y} \sim L_{\mathrm{c}}$ and $L_{z} \sim \Delta L_{z}$ may be estimated assuming that during the time interval $t_{\mathrm{d}}$ about half of the total number of pairs in the group will annihilate. For $B=B_{\mathrm{c}}$ and $\theta=\pi / 2$, we obtain $\Delta L_{z} \sim 1 \AA, t_{\mathrm{d}} \sim 10^{-17} \mathrm{~s}$ and $\Delta \mathcal{W} \sim 10^{8} \mathrm{~W}$. The total radiated power for the whole plasma bunch is given by $\mathcal{W}_{\text {tot }} \sim \Delta \mathcal{W}\left(p_{\mathrm{F}} / \Delta p_{\mathrm{F}}\right)\left(L_{z} / \Delta L_{z}\right) \sim 10^{10} \mathrm{~W}$ for $p_{\mathrm{F}} \sim m c$ and $L_{z} \sim 5 \Delta L_{z}$. 


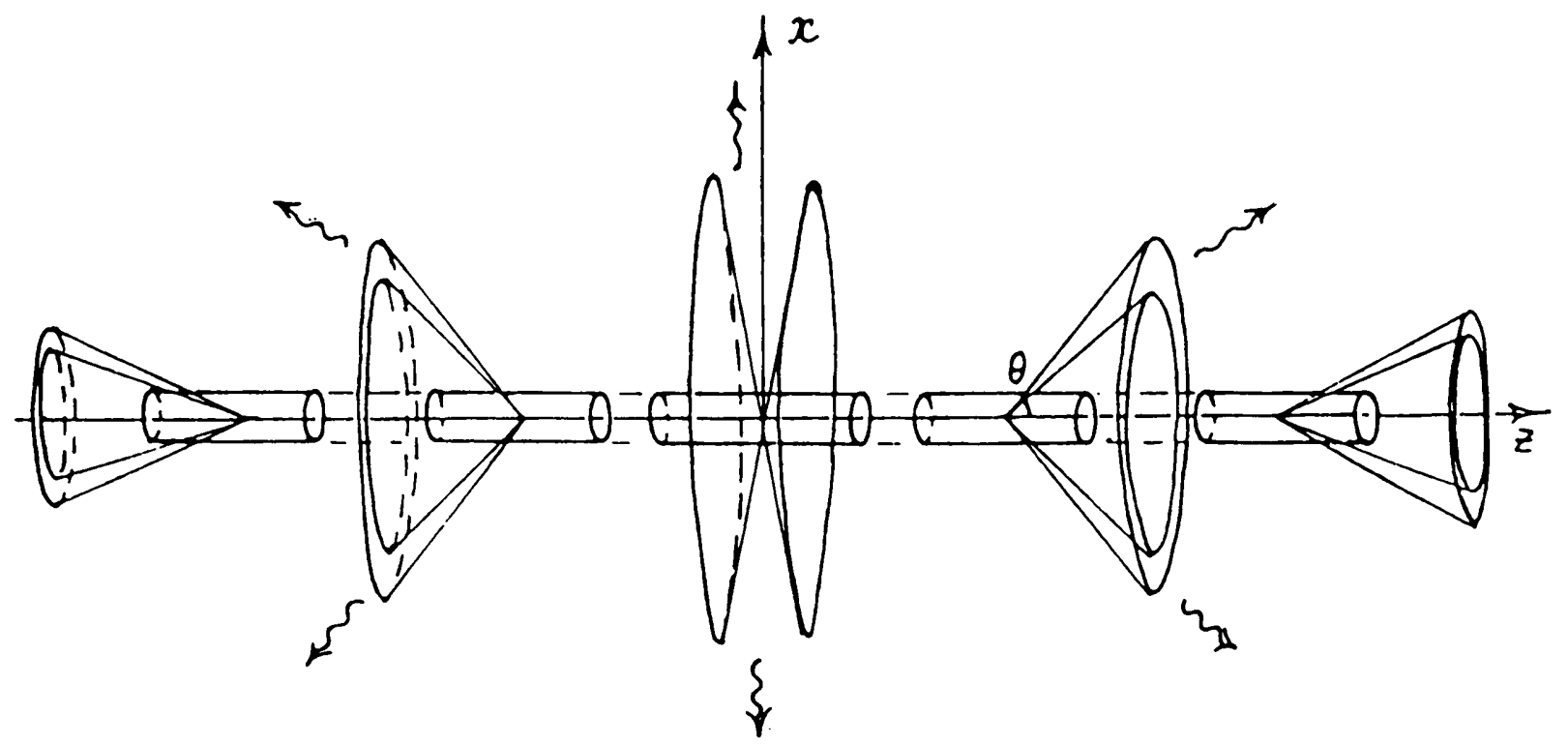

Figure 4 Geometry of the annihilation radiation of $e^{-} e^{+}$groups flying away along the magnetic field lines with different momenta $p_{\mathrm{z}}=\hbar k_{\mathrm{z}} / 2=m c \cot \theta$ at $t=t_{\mathrm{d}}$.

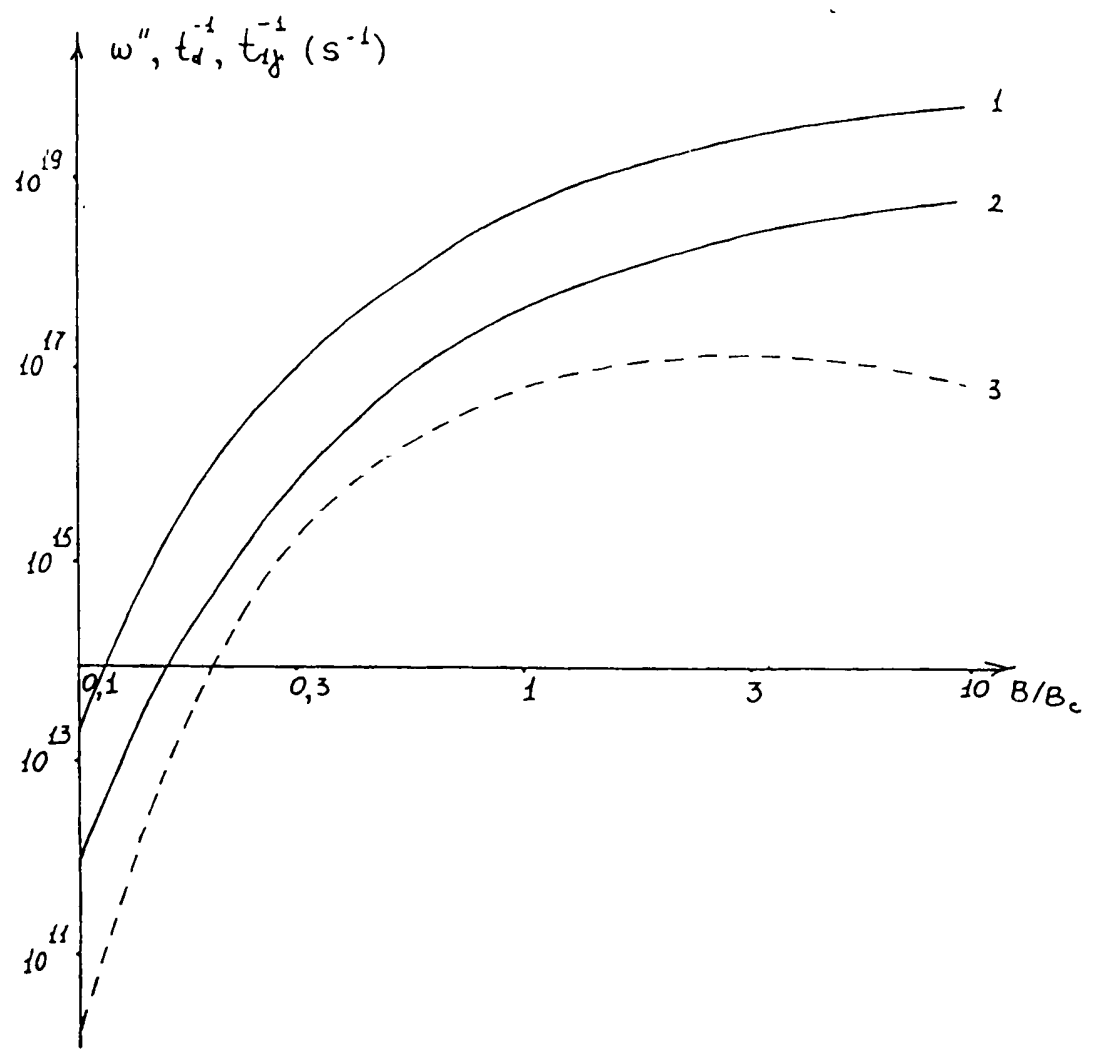

Figure 5 Growth rate $\omega^{\prime \prime}$ [eq.(5), curve 1], inverted delay time $t_{\mathrm{d}}^{-1}$ (curve 2) for $\theta=\pi / 2, s \sim L_{\mathrm{c}}$, and $\xi_{\mathrm{d}}=10$, and spontaneous one-quantum annihilation rate $t_{1 \gamma}^{-1}$ (curve 3 ) for $N_{e}=4 \times 10^{28} \mathrm{~cm}^{-3}$ vs. magnetic field strength $B / B_{c}$.

5. Finally, we have compared the CA rate with the spontaneous annihilation and collisional relaxation rates. Using the one-quantum annihilation cross-section (Wunner et al. 1986), the rate of spontaneous one-quantum annihilation may be estimated as an inverted lifetime $t_{1 \gamma}^{-1}$ of a positron with momentum $p_{z}=0$ in a totally degenerate gas of electrons, occupying the lowest Landau level. As is shown in figure 5 , for electron densities $N_{\mathrm{e}} \uparrow 10^{30} \mathrm{~cm}^{-3}$ the value of $t_{1 \gamma}^{-1}$ is much less than the CA rate. Estimation of the spontaneous the two-quantum annihilation rate, particle collisions and Compton scattering gives that for 
$B \downarrow 0.1 B_{\mathrm{c}}$ and $N_{\mathrm{e}}^{(\mathrm{s})} \uparrow N_{\mathrm{e}} \uparrow 10^{30} \mathrm{~cm}^{-3}$, these relaxation processes also cannot lead to a cancelling of the CA.

It may be of interest to discuss the fate of the annihilation $\gamma$-quanta propagating in vacuo out of an initial plasma bunch. If the external magnetic field is homogeneous and stationary, the radiated $\gamma$ quanta will remain near the annihilation resonance and convert into $e^{-} e^{+}$pairs at distances $\sim L_{\mathrm{c}}$ from the initial bunch. In this case $\mathrm{CA}$ leads to rapid and effective transverse (with respect to $B$ ) diffusion of the $e^{-} e^{+}$plasma. If the magnetic field is localized near the plasma bunch and rapidly changes at distances $\sim L_{\mathrm{c}}$ and time scales $\sim t_{\mathrm{d}}$ (e.g. when it is generated by plasma inner currents) $\gamma$-quanta may propagate without significant absorption. In these cases CA leads to the generation of coherent $\gamma$-radiation. Such a situation is discussed in Winterberg (1979) where a hypothetical experiment with collapsing electron and positron beams is described. According to Winterberg the collapse leads to $e^{-} e^{+}$plasma densities of order $10^{42} \mathrm{~cm}^{-3}$ and generation of magnetic fields $\sim 10^{16}-10^{17} \mathrm{G}$. However, at a magnetic field strength $\sim 10^{13} \mathrm{G}$ the process of CA must be taken into account. As a result only pair densities considerably lower than that mentioned in Winterberg are available.

The problem considered above proves the existence of superradiance in systems with a continuous energy spectrum. Similar to superradiance in two-level oscillators, it leads to the generation of powerful coherent radiation.

The application of the ideas developed here to similar processes in other physical situations, e.g. twoquantum annihilation of $e^{-} e^{+}$plasma, electron-ion recombination in a gas discharge, or electron-hole annihilation in semiconductors, is still a problem to be solved. As for the laboratory observation of these phenomena, semiconductors seem to offer the best possibility. In this case there would be no need for superstrong magnetic fields and tremendous plasma densities.

Acknowledgments: We thank V. V. Zheleznyakov and Yu. V. Chugunov for valuable discussions. 